\title{
High-precision, three-dimensional tracking of mouse whisker movements with optical motion capture technology
}

\author{
Snigdha Roy ${ }^{1}$, Jerí L. Bryant ${ }^{2}$, Ying Cao ${ }^{1}$ and Detlef H. Heck ${ }^{1 *}$ \\ Department of Anatomy and Neurobiology, University of Tennessee Health Science Center, Memphis, TN, USA \\ 2 Division of Genetic Medicine, Department of Medicine, Vanderbilt University, Nashville, TN, USA
}

Edited by:

Agnes Gruart, University Pablo de Olavide, Spain

\section{Reviewed by:}

Fabrício A. Pamplona, University of Cambridge, UK

Nashaat Z. Gerges, Medical College of Wisconsin, USA

\section{*Correspondence:}

Detlef H. Heck, Department of

Anatomy and Neurobiology, University of Tennessee Health Science Center,

855 Monroe Avenue, Room 405,

Memphis, TN 38163, USA.

e-mail:dheck@uthsc.edu

\begin{abstract}
The mystacial vibrissae or whiskers in rodents are sensitive tactile hairs emerging from both sides of the face. Rats and mice actively move these whiskers during exploration. The neuronal mechanisms controlling whisker movements and the sensory representation of whisker tactile information are widely studied as a model for sensorimotor processing in mammals. Studies of the natural whisker movement patterns during exploration and tactile examination are still in their early stages. Tracking the movements of whiskers is technically challenging as they move relatively fast and are very thin, particularly in mice. Existing systems detect light-beam interruptions by the whiskers or use high-speed video to track whisker movements in one or two-dimensions. Here we describe a method for tracking the movements of mouse whiskers in three-dimensions (3D) using optical motion capture technology (OMCT). OMCT tracks the movements of small retro-reflective markers attached to whiskers of a head-fixed mouse with a spatial resolution of $<0.5 \mathrm{~mm}$ in all 3D and a temporal resolution of $5 \mathrm{~ms}(200 \mathrm{fps}$ ). The system stores the 3D coordinates of the marker's trajectories onto hard disk allowing a detailed analysis of movement trajectories bilateral coordination. The described method currently uses the minimum of two tracking cameras, which requires head-fixation for reliable tracking.
\end{abstract}

Keywords: mystacial vibrissae, mouse, movement tracking, 3D tracking, whisker movement

\section{INTRODUCTION}

The rodent mystacial vibrissae system, particularly its sensory neuronal representation, have become a major focus in neuroscience (Fox, 2008). Research on the rodent whisker system has long focused on rats (Vincent, 1912) revealed a rich repertoire of movements with respect to amplitude, bilateral coordination, and modal frequency in different behavioral contexts, such as exploration and tactile examination. Several of those studies used high-speed video technology to track vibrissae movements in two-dimensions (2D; Berg and Kleinfeld, 2003; Sellien et al., 2005; Towal and Hartmann, 2006; Mitchinson et al., 2007; Grant et al., 2009). Others used linear light detector arrays (Bermejo et al., 1998; Gao et al., 2001), which need to be brought very close to the rat's head and require an attachment to the whisker to increase its diameter enough to be detected (Harvey et al., 2001). To our best knowledge, successful use of the latter method in mice has not been reported. In our hands the sensors (MX-Sensor, Metralight, Inc., San Mateo, CA, USA) were not sensitive enough to reliably track the thin mouse whiskers unless they were made thicker with an attachment that would significantly weigh them down and alter their movements. Also, the apparatus has to be positioned very close to the animals' head, which may add to the stress of head-fixation.

Compared to rats, the use of mice currently offers a much broader spectrum of genetic models for brain disorders and of neurogenetic tools to manipulate nervous system function. Mice thus offer important new opportunities to study whisker system function in the context of diseases and their potential treatments and in the context of, e.g., optogenetic manipulations of indentified cell types. In order to take full advantage of mice as an animal model for the study of the mystacial vibrissae system, efficient methods for high-precision tracking of mouse whisker movements are essential. Here we describe a method to track the movements of mouse whiskers in three-dimensions (3D) using optical motion capture technology (OMCT). OMCT derives 3D movement trajectories of retro-reflective markers from $2 \mathrm{D}$ video images captured simultaneously from different angles by two or more high-speed cameras (Abdel-Aziz and Karara, 1971). Retro-reflective elements or surfaces reflect light back to its source with a minimum scattering of light. Thus, OMCT works with relatively low light intensities.

Optical motion capture technology is widely used in biomechanical research (e.g., Blake and Ferguson, 1993) and in cinematography. Advantages of OMCT over high-speed video techniques include the facts that infrared-based OMCT does not require bright illumination and that it directly provides $3 \mathrm{D}$ trajectory coordinates including velocity and acceleration, eliminating the need for extensive post-processing.

Optical motion capture technology does, however, require attachment of retro-reflective markers onto the whiskers. But these markers can be made very small $\left(2-3 \mathrm{~mm}^{2}\right)$ and very light $(<1 \mathrm{mg})$ to minimize the impact on whisker movements.

Here we demonstrate the use of OMCT to track spontaneous movements of mouse $\mathrm{C} 4$ whiskers in $3 \mathrm{D}$. We also demonstrate that the two-camera system can be used for bilateral tracking of $\mathrm{C} 4$ whiskers on both sides of the face simultaneously and show examples of symmetrical and non-symmetrical whisker movements recorded during the same session. 


\section{MATERIAL AND METHODS ANIMALS}

C57BL/6J adult male mice were housed with food and water ad libitum in a 12:12-h light/dark cycle. All mice used in this study were raised and all experiments were performed in accordance with procedural guidelines approved by the University of Tennessee Health Science Center Animal Care and Use Committee. Principles of laboratory animal care (NIH publication No. 86-23, rev. 1996) were followed. One week prior to experiments, an aluminum head post was surgically mounted to the skull and secured with acrylic cement. The acrylic cement was anchored to the skull using three small machine screws. The aluminum post was used to fix the mouse's head to a metal holder during the experiments. A detailed description of the head-fixation procedure has been published previously (Bryant et al., 2009).

\section{MOTION CAPTURE SYSTEM}

The 3D tracking system used here (Hawk Digital Real Time System, Motion Analysis Corp., Santa Rosa, CA, USA) was equipped with the minimally required two-cameras in conjunctions with the Cortex analysis software (Motion Analysis, CA, USA; Figure 1). The X, Y, and $\mathrm{Z}$ coordinates of the whisker-attached retro-reflective markers (Figure 1B) were digitized and stored on hard drive together with video recordings of the marker movements. The markers were fashioned from a retro-reflective tape backed with adhesive (Motion Analysis Corp., Santa Rosa, CA, USA) and fastened onto the whiskers using the tape's adhesive (see below). Markers were affixed to the whisker at a distance of about $1 \mathrm{~cm}$ from the base. Reliable 3D tracking requires that a marker be visible at all times by both cameras. This condition can be satisfied in head-fixed mice where the orientation of the mouse to the cameras remains fixed. The system was connected to a dual processor Windows based computer for data collection.

Trajectory information was not available in form of real-time analog output signals that could be directly digitized with thirdparty data acquisition systems. Instead X, Y, and Z components of maker trajectories were exported off-line from the Cortex software into a spreadsheet format and then imported into a Spike2 (Cambridge Electronic Design, Cambridge, UK) data file for further analysis. To provide a temporal align for the imported trajectory components into the Spike2 data format, an analog timing signal provided by the Hawk cameras was recorded in the Spike2 data format in real-time using a CED1401 analog/digital converter (Cambridge Electronic Design, Cambridge, UK) and the Spike2 software.

\section{CAMERA SETUP AND CALIBRATION}

Cameras were positioned $90 \mathrm{~cm}$ in front and about $40 \mathrm{~cm}$ above the platform for the head-fixed mouse, separated by about $30 \mathrm{~cm}$, resulting in a viewing angle about $20^{\circ}$. The floor of the capture volume rested at an angle of $24^{\circ}$ below the camera lenses. Cameras were adjusted to create a volume viewed by both cameras (the capture volume) that contained the entire expected range of marker movements (gray box in Figure 1C). The size of the capture volume was typically in the range of $\pm 6 \mathrm{~cm}$ lateral of the midsagittal plane of the mouse and $\pm 3 \mathrm{~cm}$ above and below the head. The cameras track marker movements in real-time at 200 fps by capturing infrared light emitted from the retro-reflective marker surface. Infrared light is emitted from an array of LEDs mounted directly on the cameras, surrounding the lenses.

\section{TRACKING OF WHISKER MOVEMENTS}

Following $3-5$ days of recovery from surgery mice were trained to accept head-fixation as described previously (Bryant et al., 2009). Head-fixation involved clamping the head post to a metal holder using a setscrew.

On the days of experiments mice were head-fixed and small pieces of reflective markers with diameters of $2-3 \mathrm{~mm}$ and less than $1 \mathrm{mg}$ weight were attached to the $\mathrm{C} 4$ whisker on one or both sides of the face. We used two methods for attaching marker tape to the whiskers. In one method the tape was either directly folded over the whisker - with the adhesive side toward the

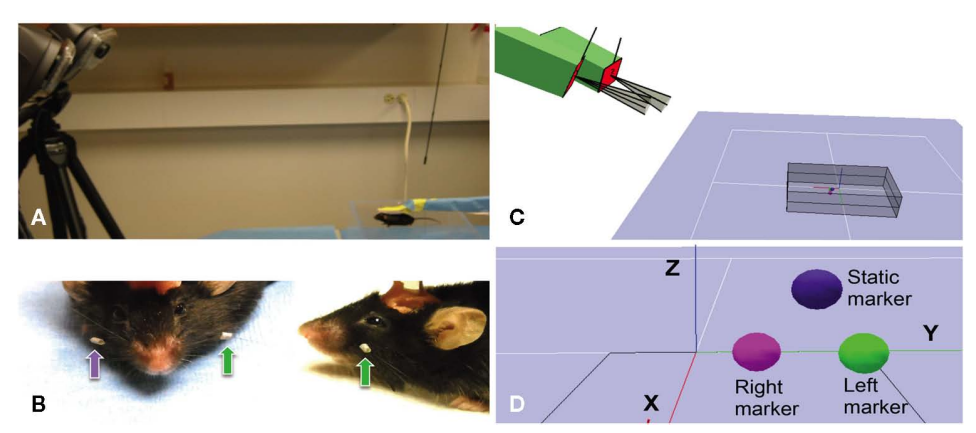

FIGURE 1 |Arrangement of tracking system, placement of whisker markers and virtual representation. (A) Photograph showing two high-speed cameras (on the left) with infrared lights are placed 0.8-1.2 $\mathrm{m}$ from the mouse (bottom right), with cameral sightlines converging on the mouse at an approximate $24^{\circ}$ angle. The mouse's head is immobilized with a head post. (B) Photographs showing close-up front and side-views of retro-reflective marker tape affixed to the left (green arrows) and right (purple arrow) C4 whisker of a C57BL/6J mouse. Acrylic cement used to mount the head post is partially visible on to of the mouse's head. (C) Screenshot from the Cortex Software during calibration showing the positions of the two-cameras (green boxes) relative to each other, outlines of their fields of view and the volume viewed by both cameras for 3D tracking. (D) Screenshot from the Cortex Software during the calibration process showing the digital representation of three retro-reflective markers, one static and two attached to the left and right $\mathrm{C} 4$ whiskers. 
whisker - using fine forceps. The second method involved covering a $2-\mathrm{cm}$ section of silicon tubing ( $1.5 \mathrm{~mm}$ outer diameter) with marker tape and cutting a 2 to $3-\mathrm{mm}$ section off the covered tubing. The short section was then slid over the whisker and secured with a small drop of Super Glue gel (3M, St. Paul, MN, USA) applied to one end of the tubing (Figure 1B). Both methods yielded similar results in terms of tracking quality. Placing the tubing on the whisker is, however, more difficult and adds slightly more weight.

Most mice will initially attempt to remove the markers until they have adapted to their presence. Reaching for the markers was prevented by taping a $1.5-\mathrm{cm} \times 5-\mathrm{cm}$ piece of cardboard in front of the forepaws. Attempts to remove markers typically ceased after 10-15 min. Movements of the C4 whiskers were recorded over several minutes.

\section{DATA ANALYSIS}

There are multiple ways of analyzing the 3D whisker trajectories, depending on the research question. Here we limited the data analysis to the velocity, acceleration, and frequency components of mouse whisker movements. Velocity and acceleration were calculated by the Motion Capture data analysis software (Cortex) as follows:

The velocity vector is calculated as a central difference for each dimension.

\section{$V_{\mathrm{x}}=$ FrameRate $*$}

$\left(\right.$ Position $_{\mathrm{X}}($ Center Frame $+i)-$ Position $_{\mathrm{X}}($ Center Frame $\left.-i)\right) /(2 * i)$

$V_{\mathrm{x}}$ is the velocity vector for the $x$-axis, Position $_{\mathrm{x}}$ the position of the marker in a specified frame and $2 * i$ is the number of frames averaged. In our experiments the frame rate was always set to $200 \mathrm{~Hz}$ and $i$ was set to 5 . Velocities for the $y$ - and $z$-axes were calculated in the same way.

The Cortex software calculates a number of motion parameters but did not provide power spectral or correlation analysis. X, Y, and $\mathrm{Z}$ coordinates of the marker trajectories can be readily exported to a spreadsheet format (Microsoft Excel). We transferred the data to Origin (OriginLab, North Hampton, MA, USA), a spreadsheet based data analysis software to generate $3 \mathrm{D}$ plots of the whisker movements and for correlation analysis of bilateral whisker movements. Correlation analysis was used to quantify the bilateral symmetry of whisker movements. Data were imported into the Spike2 software for power spectral analysis. We used power spectral density analysis and spectrogram plots to analyze and visualize the frequency components of whisker movements.

\section{RESULTS \\ WHISKING VELOCITY, ACCELERATION AND FREQUENCY}

Continuous recordings of the $3 \mathrm{D}$ trajectories of $\mathrm{C} 4$ whiskers in four different mice revealed that large amplitude rhythmic whisker movements occurred at modal frequencies of $14-16 \mathrm{~Hz}$ in each dimension (Figure 2). Along the rostro-caudal or $x$-axis, markers moved at an average maximum velocity of $435.3 \pm 167.2 \mathrm{~mm} / \mathrm{s}$ (mean $\pm \mathrm{SD}, n=5$ mice). The average maximal acceleration along the same axis was $71034.4 \pm 24001.5 \mathrm{~mm} / \mathrm{s}^{2}$ (mean $\pm \mathrm{SD}, n=5$ mice). Average maximum velocity and acceleration values for all axes are listed in Table $\mathbf{1 .}$
Table 1 | Average maximum values for velocity and acceleration ( \pm SD) of markers attached to $\mathbf{C} 4$ whiskers of five mice. Markers were attached to the whiskers at a distance of ca. $1 \mathrm{~cm}$ from the whisker base.

\begin{tabular}{lll}
\hline & Mean & 土SD \\
\hline Maximum velocity along $x$-axis $(\mathrm{mm} / \mathrm{s})$ & 435.285 & 176.212 \\
Maximum velocity along $y$-axis $(\mathrm{mm} / \mathrm{s})$ & 208.104 & 160.302 \\
Maximum velocity along $z$-axis $(\mathrm{mm} / \mathrm{s})$ & 221.005 & 93.099 \\
Maximum acceleration along $x$-axis $\left(\mathrm{mm} / \mathrm{s}^{2}\right)$ & 71034.423 & 24001.525 \\
Maximum acceleration along $y$-axis $\left(\mathrm{mm} / \mathrm{s}^{2}\right)$ & 43025.63 & 26060.12 \\
Maximum acceleration along $z$-axis $\left(\mathrm{mm} / \mathrm{s}^{2}\right)$ & 46829.746 & 23228.86 \\
\hline
\end{tabular}

\section{BILATERAL WHISKER MOVEMENTS}

Whiskers on both sides had very similar trajectories. Figure 3 shows 3D plots of the trajectories of two simultaneously tracked C4 whiskers on each side of the face from different viewing angles (Figure 3). The static images do not provide information about the dynamics and symmetry of bilateral whisker movements. As a quantitative measure for the symmetry or asymmetry of bilateral whisker movements we calculated the correlation coefficients for all three directional components of the whisker trajectory. In measurements from 3 different $\mathrm{C} 57 \mathrm{Bl} / 6 \mathrm{~J}$ mice we found average bilateral correlations of $0.113 \pm 0.246$ (mean \pm SD) for the anteriorposterior component ( $x$-axis) of the trajectories, $-0.186 \pm 0.154$ for the medio-lateral component ( $y$-axis) and $-0.206 \pm 0.427$ for the superior-inferior component ( $z$-axis).

Further analysis of bilateral correlations over shorter observation time windows $(<1 \mathrm{~min})$ revealed that the coordination of bilateral whisker movements was highly variable over time. Figure 4 shows examples of raw data and correlation results from episodes of symmetrical and asymmetrical whisker movements. Figures 4A,B shows trajectories and correlation coefficients from a period of relative symmetrical bilateral whisker movements. Correlation coefficients ranged from 0.005 to 0.156 along corresponding axes. Figures 4C,D shows data and correlation results from an episode of anti-symmetrical whisker movements. Correlation coefficients ranged from -0.1 to -0.81 along corresponding axes.

\section{DISCUSSION}

The rodent whisker system is a widely used model for the study of sensory motor processing in mammalian nervous systems. Studies of the rodent whisker system have mostly focused on rats. However, mice have become increasingly important as animals models for nervous system function because of the availability of a wide range of genetic mouse models of brain disorders and the plethora of possibilities for genetic manipulations of brain function. Mouse whiskers are thinner than those of rats and technically more challenging to track. Here we described a method based on OMCT to track mouse mystacial vibrissae or whisker movements with high spatial and temporal precision and in 3D. High-precision 3D tracking of mouse whisker movements opens up new opportunities for the detailed investigation whisking behavior and of neuronal sensorimotor processing in normal mice and mouse models of brain disorders. The 3D analysis of whisker movements has thus far received little attention and to our knowledge 

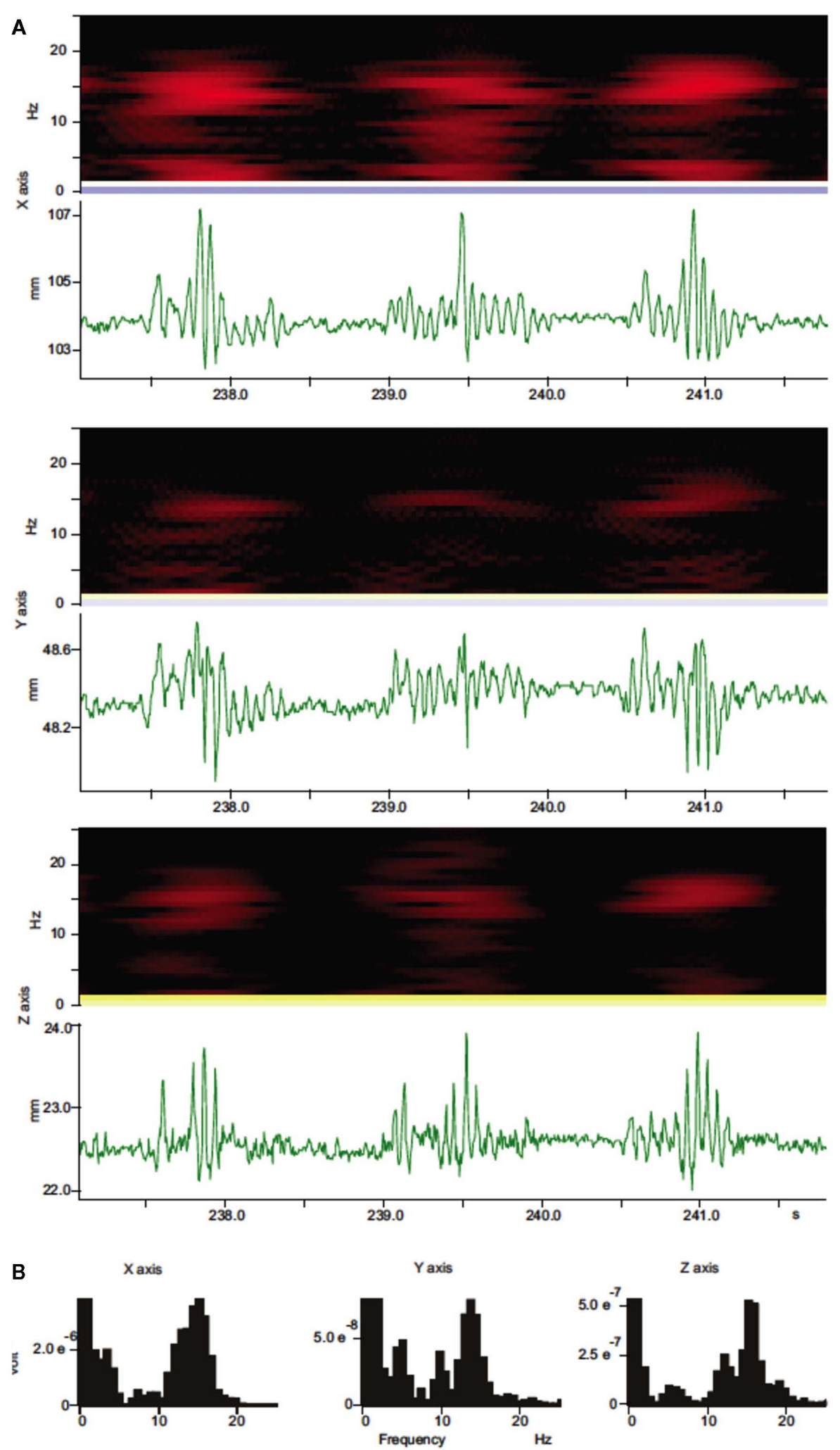

FIGURE 2 | Spectral analysis of whisker movements. (A) Spectrograms (black and red heat-maps) and trajectory components for whisker movements along each axis (green traces) are shown. Spectrograms (Hanning, block size 256) for whisker movements along all three axes show high power (bright red colors) within a stable frequency band centered around $15 \mathrm{~Hz}$ during whisker movements with variable amplitude. (B) Power spectral density histograms (Hanning, FFT size 256) calculated for the length of the trajectory components in (A) show prominent peaks around $15 \mathrm{~Hz}$. 
has never been attempted in mice. With further investigation and the establishment of baseline behavioral parameters 3D whisker analysis may prove to provide valuable and sensitive information about normal and pathological behavioral phenotypes regarding whisker use during exploration and tactile examination. Genetic, pharmacological or physiological manipulations of the neuronal network controlling whisker movements may result in subtle changes of whisking behavior that might be more easily detected in high resolution $3 \mathrm{D}$ data.

We have demonstrated that a two-camera optical motion capture system can also be used for bilateral whisker tracking. However, with only two-cameras the precise positioning of cameras becomes critical as the movements of the whiskers can cause one of the cameras to temporarily loose sight of a marker, which results in a temporary loss of 3D information. Adding a third camera would eliminate that problem in a head-fixed situation. The system used here (Hawk Digital Real Time System, Motion Analysis Corp., Santa Rosa, CA, USA) works with up to eight cameras.

In summary, tracking whisker movements with motion capture technology (OMCT) allows the easy tracking of mouse whisker movements in 3D. The method does require the attachment of retro-reflective marker tape onto the whiskers, but those markers can be made very small and very light weight to minimize impact on whisker movements. To test whether the markers had a
A

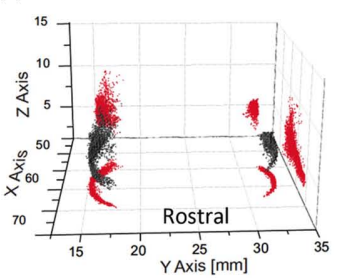

B

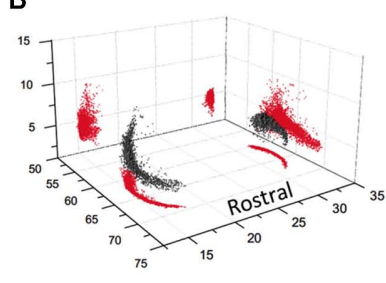

C

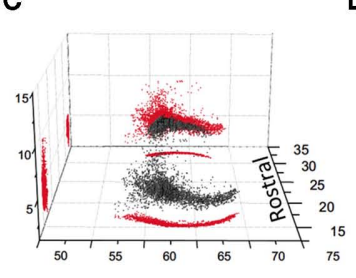

D

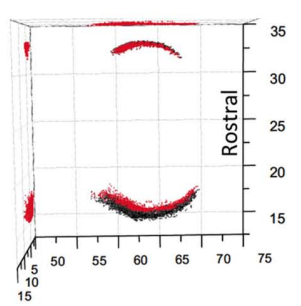

FIGURE 3 | Different viewing angles for three-dimensional (3D) reconstructions of $\mathbf{C} \mathbf{4}$ whisker movements. (A) Frontal view of the marker positions during $100 \mathrm{~s}$ (20000 frames) of whisker movements. Markers were attached to the left and right C4 whiskers. Black dots in this and all other plots represent 3D marker positions. Red dots are projections of the 3D positions onto each of the three planes. (B) Side-view of (A) rotated $35^{\circ}$ to the right. (C) Side-view of (A) rotated $90^{\circ}$ to the right. (D) Top down view (along the $z$-axis) of (C)

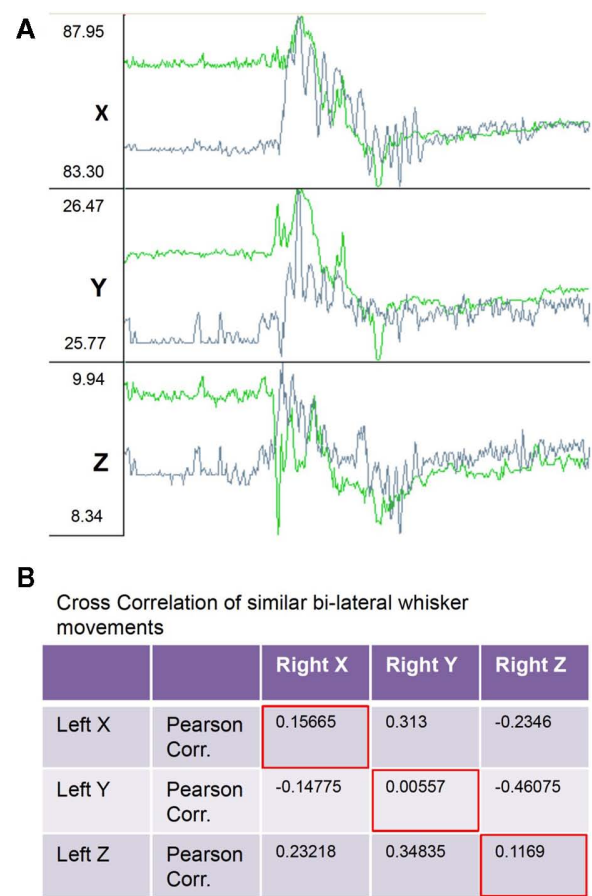

FIGURE 4 | Example raw traces of the $X, Y$, and $Z$ components of bilaterally symmetrical and non-symmetrical whisker movements. (A) Traces showing from top to bottom the $X, Y$, and $Z$ (top to bottom) components of three-dimensional movements of the left (green traces) and right (gray traces) C4 whiskers. During the 2-s (400 frames) of recordings shown the whiskers moved in a bilaterally symmetrical way. (B) Table with correlation coefficients from a correlation analysis

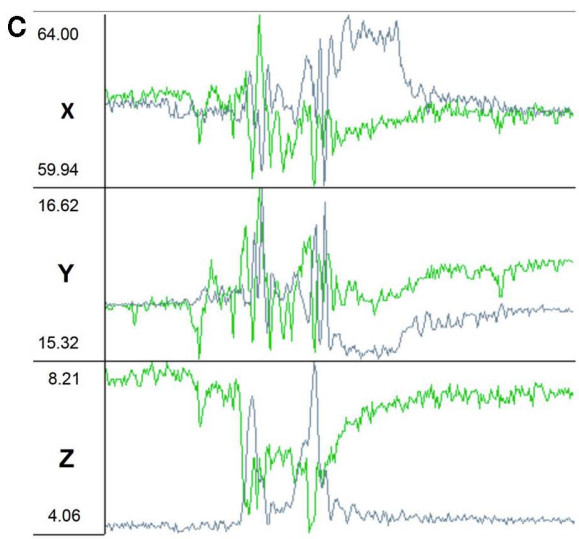

D

Cross Correlation of dissimilar bi-lateral whisker movements

\begin{tabular}{|l|l|l|l|l|}
\hline & & Right X & Right Y & Right Z \\
\hline Left X & $\begin{array}{l}\text { Pearson } \\
\text { Corr. }\end{array}$ & -0.29946 & 0.08465 & -0.35047 \\
\cline { 2 - 4 } Left Y & $\begin{array}{l}\text { Pearson } \\
\text { Corr. }\end{array}$ & -0.06668 & -0.10216 & 0.1027 \\
\hline Left Z & $\begin{array}{l}\text { Pearson } \\
\text { Corr. }\end{array}$ & -0.15561 & -0.26185 & -0.81324 \\
\hline
\end{tabular}

of $X, Y$, and $Z$ components of bilateral whisker movements shows positive correlations between corresponding components (red framed values along diagonal). (C) Traces as in (A) recorded in the same session but during a 2-s period of non-symmetrical whisker movements. (D) Correlation analysis of $X, Y$, and $Z$ components of bilateral whisker movements shows negative correlations between corresponding components (red framed values along diagonal). 
significant impact on the whisker movement we performed additional experiments using a light-beam detection device (Coulbourn Instruments, Whitehall, PA, USA) to monitor the rhythm of a single (C4) whisker's movements (i.e., intervals between the whisker interrupting the beam). We compared whisking frequencies with and without a marker attached to the whisker in three mice (No. of animal used for this experiment $n=3$ ) and found no significant changes in due to the attachment of a retro-reflective tape (mean frequency without tape: 0.0734 ; mean frequency with tape attached: 0.07251; Student's $t$-test: $t=1.16027 ; \mathrm{DF}=2 ; p=0.36572)$.

Compared to high-speed video tracking, OMCT's main advantage is that the system directly generates $3 \mathrm{D}$ trajectory, velocity, and acceleration measurements without extensive post-processing of video data. $3 \mathrm{D}$ whisking behavior in mice has not been extensively analyzed, which might at least partially be due to technical

\section{REFERENCES}

Abdel-Aziz, Y. I., and Karara, H. M. (1971). "Direct linear transformation from comparatorcoordinatesintoobject-space coordinates in close-range photogrammetry," in Proceedings of the ASP/UI Symposium on Close-Range Photogrammetry (Falls Church, VA: American Society of Photogrammetry), 1-18.

Berg, R. W., and Kleinfeld, D. (2003). Rhythmic whisking by rat: retraction as well as protraction of the vibrissae is under active muscular control. $J$. Neurophysiol. 89, 104-117.

Bermejo, R., Houben, D., and Zeigler, H. P. (1998). Optoelectronic monitoring of individual whisker movements in rats. J. Neurosci. Methods 83, 89-96.

Blake, R. L., and Ferguson, H. J. (1993). The motion analysis system for dynamic gait analysis. Clin. Podiatr. Med. Surg. 10, 501-527.
Bryant, J. L., Roy, S., and Heck, D. H. (2009). A technique for stereotaxic recordings of neuronal activity in awake, head-restrained mice. J. Neurosci. Methods 178, 75.

Fox, K. (2008). Barrel Cortex. Cambridge: Cambridge University Press.

Gao, P., Bermejo, R., and Zeigler, H. P. (2001). Whisker deafferentation and rodent whisking patterns: behavioral evidence for a central pattern generator. J. Neurosci. 21, 5374-5380.

Grant, R. A., Mitchinson, B., Fox, C. W., and Prescott, T. J. (2009). Active touch sensing in the rat: anticipatory and regulatory control of whisker movements during surface exploration. J. Neurophysiol. 101, 862-874.

Harvey, M. A., Bermejo, R., and Zeigler, H. P. (2001). Discriminative whisking in the head-fixed rat: optoelectronic monitoring during tactile detection

challenges. The method described here thus provides relatively easy access to 3D mouse whisking behavior with high spatial and temporal precision. Besides being of interest from a purely behavioral point of view, 3D data will also allow more detailed studies of the neuronal and genetic aspects of mouse whisking behavior using normal mice and mice with genetically altered nervous systems.

\section{ACKNOWLEDGMENTS}

We would like to thank Michael Nguyen for excellent custom mechanical designs and Shuhua Qi for outstanding technical assistance. This work was supported by grants from the National Institutes of Health to Detlef H. Heck (R01NS060887 and R01NS067201). The content of this publication is solely the responsibility of the authors and do not necessarily represent the official views of the NIH.

and discrimination tasks. Somatosens. Mot. Res. 18, 211-222.

Mitchinson,B.,Martin,C.J., Grant, R.A., and Prescott, T.J.(2007). Feedback control in active sensing: rat exploratory whisking is modulated by environmental contact. Proc. Biol. Sci. 274, 1035-1041.

Sellien, H., Eshenroder, D. S., and Ebner, F. F. (2005). Comparison of bilateral whisker movement in freely exploring and head-fixed adult rats. Somatosens. Mot. Res. 22, 97-114.

Towal, R. B., and Hartmann, M. J. (2006) Right-left asymmetries in the whisking behavior of rats anticipate head movements. J. Neurosci. 26, 8838-8846.

Vincent, S. B. (1912). The Function of the Vibrissae in the Behavior of the White Rat. Chicago: University of Chicago.

Conflict of Interest Statement: The authors declare that the research was conducted in the absence of any commercial or financial relationships that could be construed as a potential conflict of interest.

Received: 28 March 2011; accepted:06 May 2011; published online: 08 June 2011.

Citation: Roy S, Bryant JL, Cao Y and Heck DH (2011) High-precision, threedimensional tracking of mouse whisker movements with optical motion capture technology. Front. Behav. Neurosci. 5:27. doi: 10.3389/fnbeh.2011.00027

Copyright (C) 2011 Roy, Bryant and Heck. This is an open-access article subject to a non-exclusive license between the authors and Frontiers Media SA, which permits use, distribution and reproduction in other forums, provided the original authors and source are credited and other Frontiers conditions are complied with. 